\title{
ABRUPTIO PLACENTAE
}

A) FIBRINOGENOPENIA

B) Patologia de la Placenta y del UTero

Doctor Jorge Escobar Soto

Jefe del Departamento de Obstetricia. Facultad de Medicina. Universidad del Valle.

\section{Doctor Saulo Muñoz Delgado}

Auxiliar de Cátedra. Facultad de Medicina. Universidad del Valle.

\section{Doctor Reinaldo Mora Restrepo}

Residente de la Clínica de Maternidad de Cali.

Ha sido ampliamente reconocida la asociación clínica de la abruptio placentae, con la toxemia del embarazo. Los cambios histológicos que sufren el útero y la placenta en estas afecciones concentran la atención de los investigadores. Se busca la posible relación que exista entre las lesiones en estos órganos y la evolución clínica de este gran accidente del embarazo.

Igualmente se investiga la relación existente entre las lesiones macroscópicas de estos órganos y las lesiones microscópicas, en especial los cambios degenerativos de la fibra uterina y de la estructura de la placenta.

Hemos dirigido nuestro interés hacia la hemorragia del alumbramiento, que frecuentemente se presenta en el abruptio placentae y que sabemos se debe a la baja del nivel del fibrinógeno sanguíneo.

En la primera parte de este trabajo, que es un informe preliminar de nuestras observaciones, presentamos cuatro casos de pacientes con abruptio placentae, en quienes hemos hecho determinaciones de fibrinógeno, tratando de relacionar estos niveles de fibrinógeno con el tiempo de iniciación del cuadro de desprencimiento y la hora de evacuación del útero. 
La segunda parte se refiere a los estudios anatomopatológicos del útero de pacientes a quienes fue necesario practicarles histerectomía por lesión grave de la fibra muscular uterina y la consiguiente hemorragia por atonía. Complementamos este estudio con los hallazgos patológicos de la placenta en algunos casos de desprendimiento placentario.

\section{A) FIBRINOGENOPENIA}

Se presentan 4 casos de pacientes con abruptio placentae. En todos los casos se tomaron muestras seriadas de sangre antes y después del parto para hacer determinaciones de fibrinógeno. Todas las pacientes eran multíparas y menores de 30 años. La $€$ đad de la gestación osciló entre las 30 semanas y el término del embarazo. En dos de estas pacientes se practicó cesárea y dos tuvieron parto espontáneo. Tres pacientes ingresaron con feto muerto y la otra con feto vivo de 30 semanas de embarazo que murió a las pocas horas de su ingreso. Una presentó un cuadro ce útero de Couvelaire. No hubo ninguna muerte materna.

Se practicó el método de Greenberg para la determinación del fibrinógeno, cuyos valores normales oscilan entre 350 y 450 miligramos por ciento.

\section{CASOS CLINICOS}

Caso número 1.- $\mathrm{H}$. Cl. número 365-10-57.

A. T. de C., ama de casa, de veintinueve años, grávida 8 para 6. que ingresó a la Clínica el 24 de octubre del 57, a las 0 horas. 5 minutos, porque desde cinco y media horas antes presentaba dolores de parto y hemorragia vaginal moderada.

Al examen físico se encontró: TA.: 120/80 mm. Hg.; pulso: 90 por minuto; temperatura: 36.9 ' C. Utero de 32 centímetros de altura, hipertónico. Ruidos fetales negativos. Cuello uterino borrado $40 \%$ y dilatado 2 centímetros. Presentación cefálica flotante. Con el diagnóstico de abruptio placentae se practicó inmediatamente amniotomía y se obtuvo líquido amniótico con meconio. Se sedó a la paciente y se controló rigurosamente el trabajo de parto. Continuó con hemorragia vaginal ligera.

Parto espontáneo a las 8 horas 50 minutos de octubre 24, con feto de 2.200 gramos, nacido muerto. Alumbramiento a los 5 minutos. Coágulo retroplacentario de aproximadamente 400 gramos. La placenta presentaba un desprendimiento de aproximadamente el $\mathbf{9 0 \%}$. Pérdida de sangre durante el parto, normal. 


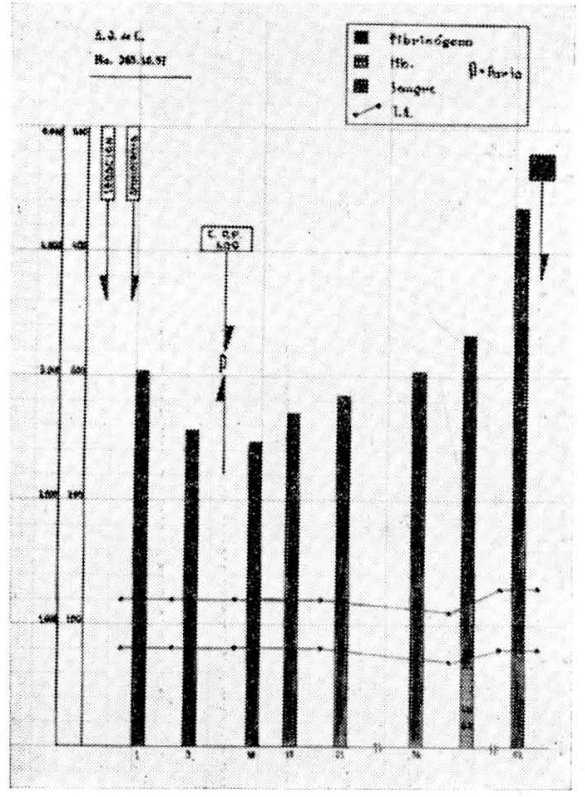

Al día siguiente de su ingreso se notó la presencia de zonas ce equimosis en los sitios donde se aplicaron inyecciones.

Desde su ingreso se tomaron muestras de sangre para la determinación de fibrinógeno con los resultados consignados en la gráfica adjunta.

El día de su ingreso y el día 26 se administraron transfusiones de sangre de 500 centímetros cúbicos. El puerperio inmediato fue normal. Se dio de alta en octubre 27, en aceptables condiciones. El eritrograma mostró severa anemia normocítica normocrómica.

Fibrinógeno: octubre 24: hora 1: $305 \mathrm{mgr} \%$; hora 5: 258 mgr.\%; hora 9: $246 \mathrm{mgr} \%$; hora 13: $270 \mathrm{mgr} \%$; hora 17: 283 mgr.\%.

Octubre 25: hora 10: $305 \mathrm{mgr} . \%$; hora 14: $329 \mathrm{mgr} . \%$.

Octubre 26: hora 8.30: $434 \mathrm{mgr} \%$.

Caso número 2.- $\mathrm{H}$. Cl. número 207-9-57.

A. T., de veintitrés años, grávida 4 para 3 , que ingresó a la Clínica en septiembre $11 / 57$, a las 10 horas, porque a las 7 horas del mismo día había iniciado trabajo de parto.

Al examen físico se encontró: TA.: 110/80 mm. Hg.; pulso: 80 por minuto; temperatura: $36^{\circ} \mathrm{C}$. Utero de 33 centímetros de 


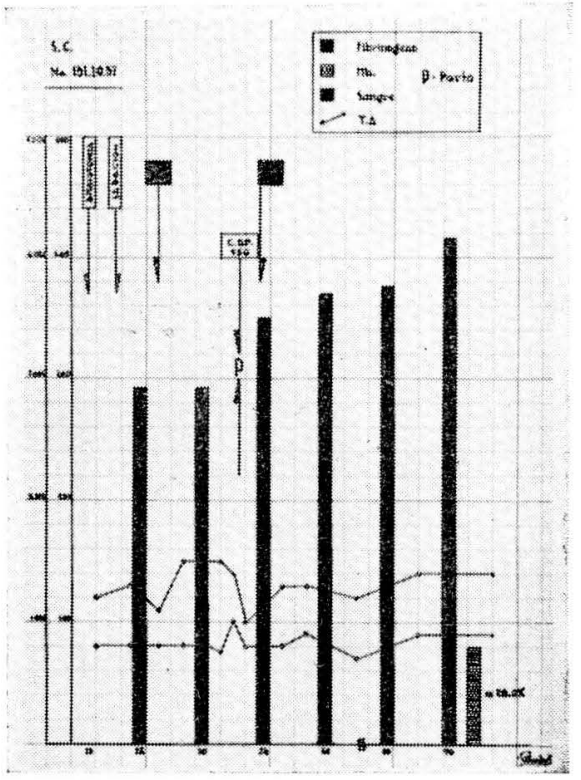

altura, hipertónico. Ruidos fetales negativos. Cuello borrado en un $30 \%$ y dilatado 1 centímetro. Presentación cefálica flotante.

Con el diagnóstico de abruptio placentae se sedó a la paciente y se practicó amniotomía a las 10 horas 55 minutos, obteniéndose líquido amniótico sanguinolento. Desde las 11 horas se tomaron muestras seriadas de sangre para determinación de fibrinógeno. Se empezó a administrar sangre total a las 11 horas 45 minutos. La altura uterina fue aumentando progresivamente y a ias 11 horas 55 minutos fue de 36 centímetros; la pérdida de sangre por vagina fue escasa. La TA. no se modificó, pero se presentó taquicardia.

En vista de la imposibilidad de obtener un parto vaginal rápido se practicó cesárea segmentaria a las 13 horas 30 minutos. La placenta presentaba la mitad de su superficie desprendida; el hematoma retroplacentario fue de 400 gramos. El niño recientemente muerto pesó 3.900 gramos. El post-operatorio fue normal. Se dio de alta en aceptables condiciones en septiembre 19. Los exámenes de laboratorio mostraron moderada anemia. La química sanguínea fue normal.

Fibrinógeno. Septiembre 11: hora 11: $370 \mathrm{mgr} . \%$; hora 14: $303 \mathrm{mgr} . \%$. 


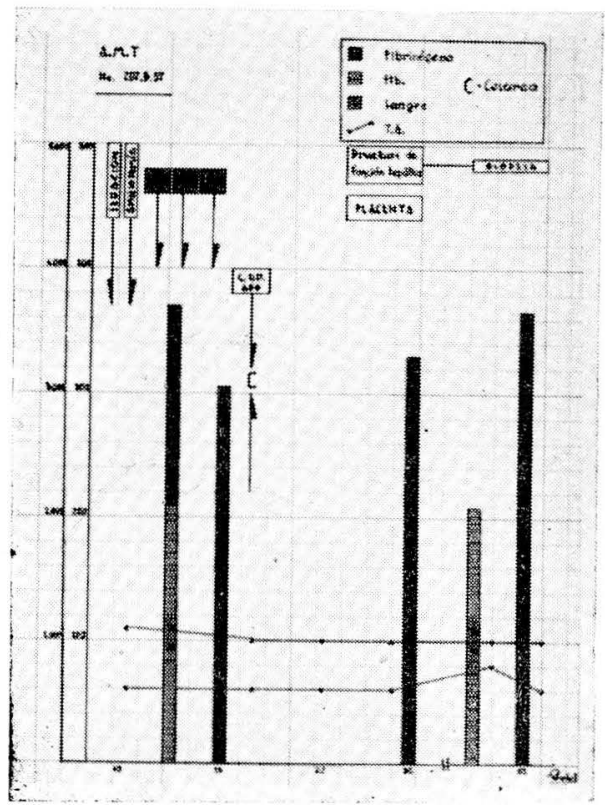

Septiembre 12: hora 8: 330 mgr.\%.

Septiembre 13: hora 7: $365 \mathrm{mgr} . \%$.

Caso número $3 .-\mathrm{H}$. Cl. 131-10-57.

S. C., grávida 15 para 11, de treinta años, que ingresó a la Clínica en octubre $8 / 57$ a las 12 horas 20 minutos, porque desde 6 horas antes presentaba dolores de parto y hemorragia vaginal escasa.

Al examen físico se encontró: TA.: 120/80 mm. Hg.; pulso: 80 por minuto; temperatura: $36.2^{\circ} \mathrm{C}$. Utero de 27 centímetros de altura, hipertónico. Ruidos fetales positivos. Cuello borrado $20 \%$ y dilatado 2 centímetros. Presentación cefálica flotante. Membranas integras. Con el diagnóstico de abruptio placentae se practicó amniotomía a las 14 horas. Se sedó a la paciente. Continuó con hemorragia vaginal escasa y la altura uterina no aumentó. A las 17 horas se inició administración de sangre. A las 17 horas 30 minutos, los ruidos fetales eran negativos.

Parto espontáneo a las 22 horas 30 minutos de octubre 8; feto de 2.000 gramos, nacido muerto. Placenta desprendida en 2/3 partes de su superficie. Coágulo retroplacentario de 750 gramos. Antes del parto se habían administrado otros 500 centímetros cúbicos de sangre. 


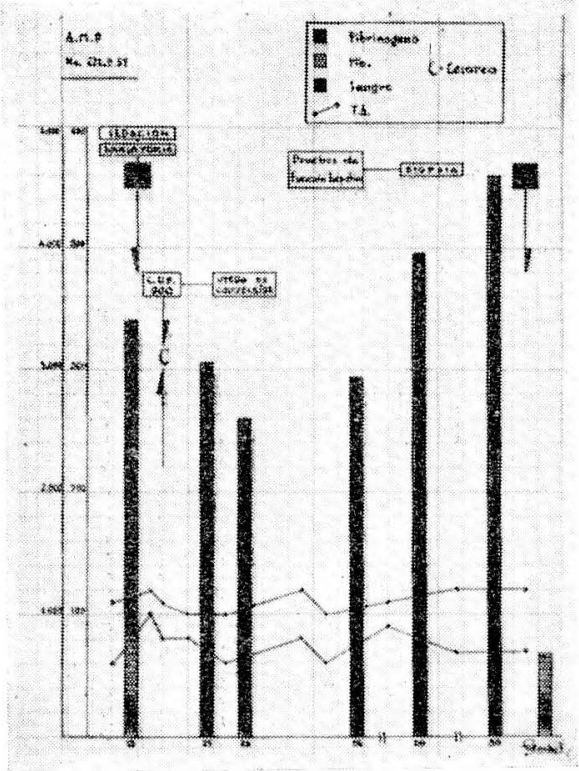

El puerperio inmediato fue normal. Se dio de alta en octubre 11/57. El eritrograma mostró severa anemia. La química sanguínea fue normal.

Fibrinógeno: octubre 8: hora 16: $296 \mathrm{mgr} \%$; hora 20: 296 mgr.\%; hora 24: 350 mgr.\%.

Octubre 9: hora 4: $350 \mathrm{mgr} . \%$.

Octubre 10: hora 7: $381 \mathrm{mgr} \%$.

Octubre 11: hora 7: $417 \mathrm{mgr} . \%$.

Caso número 4.-H. Cl. número 221-9-57.

A. M. P. de veintisiete años, grávida 5 para 4 , que ingresó a la Clínica en septiembre $11 / 57$ a las 15 horas, porque desde 3 ho:as antes presentaba dolores de parto y hemorragia vaginal escasa. Al examen físico se encontró: TA.: 110/60 mm. Hg.; pulso: 80 por minuto; temperatura: $36.5^{\circ} \mathrm{C}$. Utero de 34 centímetros de altura, hipertónico. Ruidos fetales negativos. Cuello borrado en $10 \%$ y dilatado 1.5 centímetros. Membranas íntegras, presentación cefálica flotante. Hemorragia vaginal escasa. Con el diagnóstico de abruptio placentae se practicó inmediatamente amniotomía y se sedó a la paciente.

Como no había posibilidad de obtener parto vaginal rápido se practicó cesárea transversal a las 16 horas. Se encontró útero 
cie Couvelaire, que se contrajo bien después de la inyección de pitocín-ergotrate. Feto muerto de 3.240 gramos. La placenta estaba desprendida casi en su totalidad. Coágulo retroplacentario de 800 gramos.

Previamente a la intervención y durante ésta, se administraron 1.000 centímetros cúbicos de sangre total. El post-operatorio fue normal; durante este se administraron 1.000 centíme. tros cúbicos más de sangre. El eritrograma mostró anemia severa.

Fibrinógeno: septiembre 11: hora 15: $339 \mathrm{mgr} \%$; hora 21: 305 mgr.\%; hora 24: 260 mgr.\%.

Septiembre 12: hora 10: $293 \mathrm{mgr} . \%$.

Septiembre 13: hora 11: $390 \mathrm{mgr} \%$.

Septiembre 14: hora 7: $434 \mathrm{mgr} \%$.

En septiembre 21 se dio de alta en aceptables condiciones.

\section{RESULTADOS}

Las determinaciones seriadas de fibrinógeno en el plasma, mostraron hipofibrinogenemia en todos los casos, la que se acentuó progresivamente hasta el vaciamiento del útero, a pesar de haberse administrado sangre total.

Inmediatamente después de la evacuación uterina el valor del fibrinógeno empezó a ascender en 3 de los casos. En el caso que presentó útero de Couvelaire, el valor del fibrinógeno aún continuaba descendiendo 10 horas después del vaciamiento del útero y 19 horas después de éste era inferior del valor encontrado a su ingreso.

Expresado en porcentaje el descenso de los valores de fibrinógeno desde la cifra obtenida a su ingreso, hasta el descenso máximo que se produjo en el momento del parto, se obtuvo para el primer caso 20\%; para el segundo caso, 19\%; para el tercer caso $0 \%$, a pesar de haberse administrado sangre. En el caso número 4, a las 10 horas después del vaciamiento continuaba descendiendo y el valor obtenido fue del $24 \%$ inferior al de su ingreso. A las 19 horas este valor aún era $14 \%$ inferior al de su ingreso. Los valores de fibrinógeno que se hubieran encontrado seguramente hubieran sido menores, si no se hubiese practicado amniotonía prematura y administración de sangre total. En uno de los casos se presentó equimosis en los sitios donde se aplicaron inyecciones. 
La determinación del fibrinógeno en sangre citratada conservado 2 o 3 días en nuestro Banco de Sangre mostró un promedio de $178 \mathrm{mgr} \%$.

\section{COMENTARIOS}

En tres de los casos, el valor del fibrinógeno descendió hasta el momento del parto y empezó a ascender inmediatamente después de éste, exceptuando uno de ellos que presentó útero de Couvelaire, en que, a las 10 y 19 horas después del vaciamiento era inferior en un $24 \%$ y un $14 \%$ respectivamente al valor hallado a su ingreso. Por estos hallazgos se nota la influencia que tiene el tiempo transcurrido desde la iniciación del abruptio placentae hasta el vaciamiento uterino. También la influencia del contenido uterino como productor de una sustancia que disminuye la tasa de fibrinógeno.

En el caso en que se presentó útero de Couvelaire, el contenido de fibrinógeno continuó descendiendo varias horas después del parto posiblemente debido a la reabsorción de la sustancia ciefibrinizante de la pared uterina y anexos.

En todos los casos, a pesar de la administración de sangre total, la cifra de fibrinógeno continuó descendiendo.

\section{HALLAZGOS MICROSCOPICOS EN ABRUPTIO}

M-55-1.044.-Histerectomía. La pared del útero muestra extensas áreas de hemorragia. La placenta muestra pequeñas áreas cel infarto isquémico.

M-55-1.125.-Histerectomia. Hay hemorragias intersticiales que son más notorias en la porción media de la pared uterina. Hay una reacción inflamatoria aguda en la decidua basal; las arterias helicoidales muestran una ligera endarteritis. Placenta a término que presenta áreas focales de calcificación. Hay pequeñas áreas de hemorragia subamniótica y algunas arterias vecinas muestran inflamación de la pared.

M-57-2.472.-Histerectomía. En la parte que corresponde a segmento, hay hemorragia intersticial. En el cuerpo uterino, placenta y cordón no se observa nada anormal.

M-56-189.-Histerectomía. Se observan zonas hemorrágicas intramiometriales deciduales en diferentes partes del útero. En uno de los ovarios se encuentra un cuerpo amarillo.

M-55-1.413. - Se observa una placenta a término que presenta áreas focales de calcificación. Hay pequeñas áreas de hemo- 
rragia subamniótica y algunas arterias vecinas muestran inflamación de su pared. Infarto D. Ruptura de capilares.

M-55-1.603.-Las preparaciones muestran fragmentos de placenta en los cuales las vellosidades coriales son excesivamente delicadas y atróficas y tienen muy pocos vasos. El corión es edematoso. Infarto C. Zonas de hialinización; acúmulos de fibrina. Zonas de calcificación.

M-56-16.-Infarto reciente. Zonas hemorrágicas intervellosas. Vellosidades jóvenes (24 semanas), que no alcanzaron a sufrir trastornos patológicos.

M-56-51.-Placenta envejecida con un discreto proceso de arterioesclerosis. Infarto D. Algunas vellosidades ensanchadas con desaparición del espacio intervelloso. Algunas zonas con aumento del número de vellosidades por campo.

M-56-153.-La estructura es aparentemente normal. En el cordón se observan los vasos sanguíneos muy dilatados. Infarto C.

M-57-259. - Se observan fragmentos de placenta a término, con vellosidades maduras, conglomerado nuclear de las células ciel trofoblasto y algunos depósitos de fibrina. Infarto A. Depósitos de fibrina subcoriónico. Hemorragia intervellosa.

M-57-1.016.-Las vellosidades son pequeñas con trofoblasto poco activo como corresponde a una placenta a término. Algunas vellosidades ensanchadas. Dilatación capilar.

M-57-3.237.-Vellosidades coriales pequeñas con escaso trofoblasto vascularizado. Presenta numerosos acúmulos de fibrina, algunos hialinizados. Zonas de infarto reciente.

\section{B) PATOLOGIA DEL UTERO Y LA PLACENTA}

Para complementar este estudio presentamos los principales hallazgos anatomopatológicos de la abruptio placentae y 4 casos, en los que practicaron histerectomia por atonía uterina a consecuencia de útero de Couvelaire con degeneración de las fibras musculares. Adiemás el estudio de los hallazgos anatomopatoló_ gicos en placentas de casos típicos de desprendimiento prematuro de placenta.

Se ha demostrado que los cambios patológicos del abruptio pueden ser determinados tanto macroscópica como microscópicamente en las placentas. La diferencia de estas lesiones con las de la toxemia varían solamente en intensidad: en el abruptio son más extensas. 
Los cambios histológicos del útero, descritos por Couvelaire, determinan la conducta quirúrgica en los casos asociados a gran degeneración de la fibra muscular. Sin embargo, la patología uterína no está en relación con la extensión del desprendimiento, ni. con el tamaño del coágulo retro-placentario, pues hemos encontrado casos típicos de "útero de Couvelaire", con gran estravasación sanguínea, que han evolucionado en forma normal, con buena retracción uterina.

Esto nos permite afirmar que los cambios microscópicos no están en relación con los macroscópicos, y que la conducta en casos de cesárea está supeditada a la observación de la retracción del útero (número 221-9-57).

En un caso se practicó cesárea con diagnóstico de abruptio, al no lograr la evolución espontánea del parto. Presentó intensa hemorragia, shock, aumento del tamaño del útero, dolor, hipertonía, etc. Los hallazgos macroscópicos nos inclinaban a practi_ car histerectomía ya que encontramos hemorragias subserosas que prácticamente habían disecado el peritoneo visceral, gran hemorragia del músculo uterino, hematomas disecantes de los ligamentos anchos en cantidad aproximada de unos 500 centimetros cúbicos y en general cambios tan grotescos que prácticamente habían hecho desaparecer los límites de los órganos. Sin embargo, 10 minutos después de la aplicación de pitocín y ergotrato, después de la cesárea segmentaria, se consiguió una retracción satisfactoria, que nos permitió asegurar su recuperación, como evidentemente sucedió. Como este caso, hemos tenido algunos en la práctica privada, donde hemos seguido una conducta espectante, con buenos resultados. También podemos aseverar, para confirmar, el hecho de que el aspecto macroscópico no está en relación con los cambios histológicos, en la observación de una paciente que presentó un cuadro de afibrinogenemia- severa asociada a una atonía uterina, después de un abruptio placentae. Se hizo necesaria la histerectomía por la inercia. Su aspecto macroscópico era realmente benigno, pues solamente encontramos dos áreas pequeñas de hemorragia subserosa en el cuerpo uterino y un segmento hemorrágico y friable, que nos hizo difícil la sutura del muñón vaginal. A los cortes microscópicos de la porción segmentaria, se encontraron zonas de hemorragia y necrosis, que fueron la causa de la atonía uterina por degeneración de la fibra segmentaria (M-57-2.472).

Otros observadores han anotado la desproporción entre la lesión patológica y el cuadro clínico. En casos en donde se han pro- 
ciucido pequeños desprendimientos placentarios han encontrado cuadros de "útero de Couvelaire" y al contrario en casos con grandes hematomas placentarios, úteros aparentemente normales.

Aspecto macroscópico.-Al abrir la cavidad abdominal se puede apreciar en algunos casos severos, exudado sero-sanguinolento en cantidad variable. Así mismo, en estas pacientes se en. cuentran hemorragias que han infiltrado hasta el peritoneo parietal anterior, posterior y vesical.

Se aprecian hemorragias peritoneales sub-serosas difusas o circunscritas, que le dan una apariencia peculiar, cuyo aspecto es ae todos conocido. Los tejidos adyacentes como el peritoneo visceral y parietal, los ligamentos anchos y redondos, las trompas y los ovarios, a menudo participan en el cuadro. Todo este conjunto tiene coloraciones diferentes que varían de un rojo oscuro a violáceo.

Los hematomas y la sufusión hemorrágica son tan intensos, que por sí solos explicarían la anemia de la paciente.

Si el cuadro macroscópico no está en relación con la patología microscópica, nosotros sí hemos observado la relación entre éste y el estado general y tóxico que presenta la paciente.

En ocasiones la serosa visceral se halla afectada por fisuras, posiblemente consecutivas a la ruptura por la estravasación sanguínea. Además es de una friabilidad enorme que hace complicado su manejo, y en ocasiones difícil lograr la sutura. El tamaño ciel útero se halla casi siempre aumentado considerablemente, con paredes engrosadas, reblandecidas y hemorrágicas. En los cortes de la pared en los casos de cesárea, se ha encontrado que sale líquido sero-sanguinolento, hecho que para unos autores es interesante, pues creen que es posiblemente líquido amniótico o suero sanguíneo que se halla a presión, y que ha sido forzado a través de los vasos uterinos cuando la presión intrauterina exceda la presión dentro de los sinusoides venosos. Esta es una de las teorías mecánicas sobre la forma de pasar productos tóxicos al torrente sanguíneo materno.

En 4 casos en que hemos practicado histerectomía por lesión de la fibra muscular, se han encontrado cambios histológicos característicos (M-55-1.044 y M-55-1.125).

Los haces musculares se hallan disociados por la hemorragia intersticial, que en uno de ellos era más notoria en la porción media de la pared uterina. En ocasiones se ven como si los haces estuvieran nadando en sangre estravasada. 
Holmes ha encontrado amplia infiltración leucocitaria en los tejidos afectados y los polinucleares casi en igual número que los eritrocitos.

Los vasos sanguíneos muestran varios cambios, tales como engrosamiento de las paredes y distensión, a la par que otros pueden estar vacíos.

Las reacciones deciduales son frecuentes, y en uno de nuestros casos de histerectomía se encontró reacción inflamatoria aguda en la decidua basal. Las arterias helicoidales mostraron ligera endarteritis (M-55-1.125). Para Holmes, los vasos del útero $y$ de la placenta muestran engrosamiento de sus paredes y en casos llegan a la oclusión de su luz. Uno de los casos presentó infartos isquémicos (M-55-1.044).

Aspecto macroscópico de la placenta.-El aspecto macroscópico depende en gran parte del tiempo de evolución de la separación, pues entre mayor sea éste se producirán mayores cambios en el aspecto de la placenta.

La superficie materna está cubierta por grandes coágulos, que en ocasiones se logran desprender con facilidad, pero generalmente dejan una zona escavada o deprimida dentro del tejido placentario. Esta depresión será mayor, cuanto más tempranamente, durante el parto, se haya presentado el desprendimiento. Así mismo, esta superficie estará más cubierta de sangre y coágulos, en proporción al grado de separación (números 221-957 y 207-9-57).

Las características del coágulo dependen de la severidad y localización de la hemorragia. En los casos en que la separación se inicie en la parte central de la placenta, el hematoma será mayor que en aquellos en los cuales la separación se inicia marginalmente, así como también la separación será más completa a medida que aumente el hematoma. Este mecanismo de iniciación podríamos compararlo a los métodos de desprendimiento de la placenta durante el alumbramiento; comenzando por el centro como una sombrilla que se invirtiera, o desde el borde proseguir hacia la parte central.

En el caso de la separación por la parte marginal, la sangre diseca más fácilmente las membranas abriéndose paso al exterior, y por lo tanto se producirá una menor separación. De esta manera la severidad del abruptio no lo podemos medir por la cantidad de hemorragia externa, sino sobre las reacciones generales que produzca por la absorción de materiales tóxicos y por 
la anemia producida por la pérdida sanguínea total, tomando en cuenta la pérdida oculta.

Fish J. S., opina que los trombos vasculares pueden ser mirados como un mecanismo de protección que tiene la finalidad ce prevenir posteriores absorciones de este material tóxico dentro del torrente sanguíneo materno. Nosotros no compartimos este punto de vista, pues hemos observado que a mayor tiempo entre la producción del abruptio y el parto, mayor será la desfibrinación de la sangre y, por consiguiente, el shock, la hemorragia y demás fenómenos tóxicos.

Este es, a grandes rasgos, el aspecto macroscópico, pero nuestra experiencia nos ha demostrado que es difícil determinar la extensión de área infartada hasta estudiar la placenta después cie dos semanas de fijación en formol. Así se logra que grandes zonas con hemorragia, que hacian indiferenciable el campo, desaparezcan y se delimiten las zonas infartadas. Estas se verán de coloración casi negruzca en oposición al tejido sano que tiene un color claro y cuya consistencia es más firme que el tejido que lo rodea.

Fish opina que la fijación de la placenta ofrece las siguientes ventajas:

1a Permite seccionar adecuadamente y exponer claramente el tejido placentario.

$2^{\text {a }}$ Evita que la superficie se cubra de sangre, lo que no permite un buen examen.

$3^{\text {a }}$ Previene el drenaje del sistema fetal y el colapso completo del sistema intervelloso, y por lo tanto conserva una relación más aproximada a lo normal.

4a Realza ciertos cambios característicos en las áreas afectadas en cuanto al color, consistencia y forma, cambios que son imposibles de apreciar en las placentas frescas, pero que se hacen evidentes después de su fijación.

5a Permite una selección fácil y segura para el estudio mi_ croscópico de las áreas representativas.

Creemos que ésta fue una de las fallas que tuvimos al prin. cipio para identificar las lesiones, por la cual tuvimos que deshechar más de 100 ejemplares. Bartholomew, siguiendo esta técnica ha encontrado un cuadro patognomónico en las toxemias y ha llegado al diagnóstico en la mayoría de los casos a partir de una placenta de paciente desconocida. 
Hemos practicado los cortes en secciones paralelas resultando pequeñas tiras de tejido placentario donde hemos identificado los infartos como enclavados en el tejido placentario.

En los casos de abruptio placentae, hemos encontrado infartos agudos. La localización de estos infartos es casi siempre central, circunscritos y en ocasiones se extienden ocupando hasta $2 / 3$ partes del tejido placentario.

La zona infartada presenta una superficie granular que no refleja la luz, rodeadas por áreas de color más claro y brillantes.

Aspecto microscópico.-El tipo más frecuente de infarto asociado con abruptio placentae, son los descritos por Bartholomew como infartos $\mathrm{D}$ y $\mathrm{E}$, que son mucho más agudos que los otros infartos y que se localizan en cualquier parte de la placenta, pero con más frecuencia en la superficie materna o dentro de la placenta. Su etiología parece debida a la oclusión o trombosis de una pequeña arteria placentaria. El color negro es debido a la detención de la sangre venosa en las vellosidades congestionadas, cuyos capilares se hallan distendidos o rotos. Las vellosidades muestran distención de la mayoría de los capilares, encontráncose con abundante cantidad de eritrocitos que en ocasiones sobrepasan los 20 .

Bartholomew opina que la ruptura de los capilares de las vellosidades forman coágulos que le dan la apariencia oscura.

La necrosis de las vellosidades ocurre más rápidamente que en los demás infartos y es por esto que las vellosidades fijan menos el colorante, muestran mayor degeneración y alguna desintegración.

Microscópicamente hay una zona de demarcación entre el área infartada y el resto del tejido placentario, que se ve más claro con vellosidades de tamaño normal, capilares pequeños y con estructuras nucleares normales.

Fish ha encontrado, en las áreas oscuras, los siguientes cambios que son evidentes: 10 Las vellosidades están ensanchadas hasta tal punto, que llegan a ocluir el espacio intervelloso; $2 \%$ Los capilares vellosos están marcadamente distendidos y en ocasiones se ven ocupados casi totalmente e ingurgitados por eritrocitos, cuya salida está impedida debido a la obstrucción venosa; 3 ? Son aparentes los signos de necrosis en los núcleos de las células de las vellosidades encontrándose picnosis, cariorrexis y cariolisis cuyo avance dependerá de la edad y agudeza de la lesión. 
Los infartos clasificados como D y E, por Bartholomew, pueden ser típicos del abruptio, y su diferencia estriba en que el infarto D es una forma menos aguda del $\mathrm{E}$ y que muestra completa desintegración nuclear y degeneración de las vellosidades.

\section{BIBLIOGRAFIA}

BARTHOLOMEW, R. A.; COLVIN, E. D.; GRIMES, W. H.; FISH, J. S. Am. J. Obs. and Gynec. 74: 1, 1957.

BURSTEIN, R.; BLUMENTHAL, H. T.; SOULE, S. D.-Am. J. Obs. and Gynec. 74: 1, 1957.

CORREA PELAYO-1956, Cali. Patología de las Toxemias (en prensa).

DARO, A. F.; GOLLIN, H. A., NORA, E.-Am. J. Obst. and Gynec. 72: 3, 1956.

DIECKMANN W. J.-The Toxemias of Pregnancy, 1952.

DUCHAINE, P.-Am. J. Obst. and Gynec. 73: 6, 1957.

FISH, J. S.-Hemorrhage of late pregnancy, 1955.

HODGKINSON, C. P.; LUZADRE, J. H.; PIFER P. W.-Obst. and Gynec. 5: 4, 1956.

KINCH, R. N.-Am. J. Obst. and Gynec. 71: 4, 1956.

LEWIS, L. T. T.-Progress in Clinical Obstetrics and Gynecology, 1956.

MUNOZ D. SAULO-1956. Cali. Complicaciones de las Toxemias del Embarazo.

MURPHY, C. J.; PICOT, H.; THOMPSON, H. G.-Am. J. Obst. and Gynec. 72: 6, 1956.

THEOBALD, G. W.-The Pregnancy Toxemias, 1956. 